This is an electronic reprint of the original article. This reprint may differ from the original in pagination and typographic detail.

Author(s): Valkonen, Arto; Chukhlieb, Maryna; Moilanen, Jani; Tuononen, Heikki; Rissanen, Kari

Title: $\quad$ Halogen and Hydrogen Bonded Complexes of 5-lodouracil

Year: $\quad 2013$

Version:

Please cite the original version:

Valkonen, A., Chukhlieb, M., Moilanen, J., Tuononen, H., \& Rissanen, K. (2013). Halogen and Hydrogen Bonded Complexes of 5-lodouracil. Crystal Growth and Design, 13(11), 4769-4775. https://doi.org/10.1021/cg400924n

All material supplied via JYX is protected by copyright and other intellectual property rights, and duplication or sale of all or part of any of the repository collections is not permitted, except that material may be duplicated by you for your research use or educational purposes in electronic or print form. You must obtain permission for any other use. Electronic or print copies may not be offered, whether for sale or otherwise to anyone who is not an authorised user. 


\title{
Halogen and Hydrogen Bonded Complexes of 5-Iodouracil
}

\author{
Arto Valkonen, ${ }^{\dagger}$ Maryna Chukhlieb, ${ }^{\dagger}$, Jani Moilanen, $^{\ddagger}$ Heikki M. Tuononen, ${ }^{\ddagger}$ and Kari \\ Rissanen ${ }^{*} \dagger$ \\ $\dagger^{\dagger}$ Department of Chemistry, Nanoscience Center, University of Jyväskylä, P.O. Box 35, 40014 \\ Jyväskylä, Finland \\ $\$$ Department of Chemistry, University of Jyväskylä, P.O. Box 35, 40014 Jyväskylä, Finland
}

\begin{abstract}
Three derivatives of 5-iodouracil were prepared and their complexation properties, supplemented by 5-iodouracil under the same conditions, studied with and without halogen bond acceptors in $N, N$-dimethylformamide, $N, N$-diethylformamide, $N$-methylformamide, formamide, dimethylsulfoxide, and water. The intermolecular halogen and hydrogen bonding interactions observed in the solid state were investigated using single crystal X-ray diffraction and quantum chemical calculations, and the acquired data were contrasted with bonding interactions previously reported for 5-iodouracil in the Cambridge Structural Database. It was found that the polarized iodine atom and the amidic NH functionality act simultaneously in 5-iodouracil and its derivatives, for which reason the halogen and hydrogen bonds play an equally important role in controlling the resulting crystal structures.
\end{abstract}

* Corresponding author:

Kari Rissanen

Tel.: +358505623721

E-mail: kari.t.rissanen@jyu.fi

https://www.jyu.fi/kemia/tutkimus/orgaaninen/staff/rissanen 


\section{INTRODUCTION}

5-Iodouracil (5IU) is a simple and commercially available pyrimidine nucleobase derivative in which the alkenyl hydrogen closest to the carbonyl group of the uracil moiety is substituted by iodine. This system, along with other 5-halouracils and their derivatives, has been of particular interest due to its biological, viz. antitumor and antiviral, activity. ${ }^{1}$

The first crystal structure with a 5IU unit (5-iodo-2'-deoxyuridine) was reported already in $1965,{ }^{2}$ and the online portal to the Cambridge Structural Database (WebCSD) ${ }^{3}$ contains two separate reports of the same monoclinic structure. ${ }^{4}$ The iodine atom in 5IU is bonded to a $s p^{2}$ hybridized carbon and becomes polarized due to the adjacent electron withdrawing carbonyl group. Accordingly, 5IU should manifest itself as a halogen bond (XB) donor, but, to our knowledge, the experimental evidence is limited to some biological systems. ${ }^{5,6}$ Of late, halogen bonding has attained increasing interest by many research groups, as discussed in several reviews dealing with XBs in the solid state, ${ }^{7}$ in solution, ${ }^{8}$ and in biological systems. ${ }^{9}$ Recently, the first cases of improved pharmaceutical properties effected/induced by halogen bonding were demonstrated. ${ }^{10}$

Despite the potential of 5IU framework for crystal engineering, the number of structural entries in the CSD that contain this unit is surprisingly small: only 26 entries can be found and most of them are derivatives of uridine which is a nucleoside with a ribose ring attached to the uracil ring via a $\beta$-N1-glycosidic bond. However, even when examining this limited set of data, it became clearly evident from the range of donor-acceptor bond lengths observed that 5IU could act as a $\mathrm{XB}$ donor in different systems. Inspired by this, and due to our own interest on alternative XB donors (i.e. donors in which the halogen atom is not polarized by fluorine), ${ }^{11}$ we decided to conduct a thorough investigation of halogen bonding with 5IU. Consequently, crystallization 
experiments involving $5 \mathrm{IU}$ and its three $N$-substituted derivatives were performed in suitable solvent systems containing electron donors such as $\mathrm{N}, \mathrm{N}$-dimethylformamide (DMF), $\mathrm{N}, \mathrm{N}$ diethylformamide (DEF), $N$-methylformamide (MeF), formamide (FA), dimethylsulfoxide (DMSO), and water. We also performed quantum chemical calculations for simplified model systems as well as co-crystallization attempts with 5IU and its derivatives using selected organic molecules and salts having capabilities as XB acceptors. While the focus of the current work was on the XBs exhibited by the 5IU unit, the examined systems also display a number of hydrogen bonds (HBs) that are included in the discussion. 


\section{EXPERIMENTAL SECTION}

\section{Materials}

5-Iodouracil (5IU, 1) was obtained from Aldrich (98\% purity) or from Alfa Aesar (97 \%) and used as such. Benzyl bromide, 1-bromohexane, and (2-bromoethyl)trimethylammonium bromide were obtained from Fluka ( $>98 \%$ ), Acros ( $>99 \%$ ), and Aldrich (98\%), respectively, and used without further purification. Potassium carbonate was obtained from Merck $(>99 \%)$ and dried in an oven before use. All other chemicals were obtained from commercial sources and used as such. Organic solvents used in crystallizations and syntheses were dried before use by standing over $3 \AA$ molecular sieves. The compounds prepared and investigated in this study (1-4) are presented in Scheme 1.

Scheme 1. Schematic structures of compounds 1-4 including the atom numbering schemes.

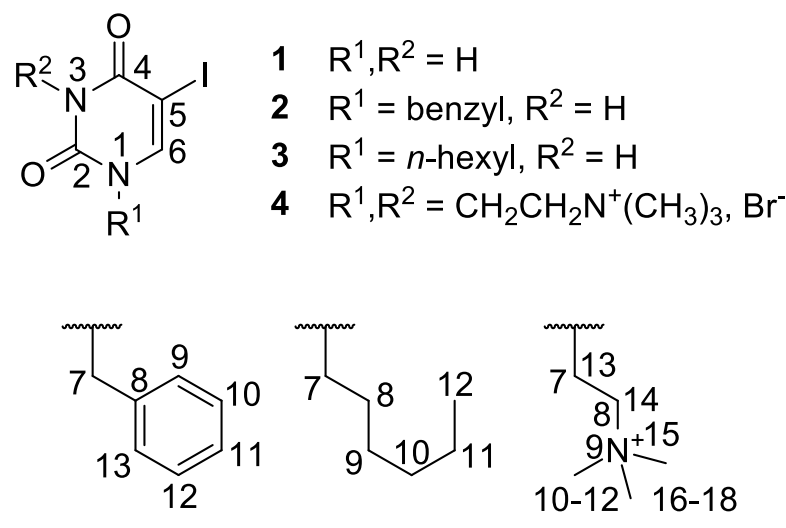

\section{Synthesis of derivatives of 5IU}

Compound 2 (1-benzyl-5-iodopyrimidine-2,4(1H,3H)-dione): The preparation of 2 was started by dissolving $1(0.92 \mathrm{~g}, 3.86 \mathrm{mmol})$ and $\mathrm{K}_{2} \mathrm{CO}_{3}(0.54 \mathrm{~g}, 3.86 \mathrm{mmol})$ in dry DMF $(10 \mathrm{ml})$ in a round-bottomed flask. The reaction mixture was stirred and heated mildly (at $45^{\circ} \mathrm{C}$ ) under $\mathrm{N}_{2}$ 
atmosphere for $30 \mathrm{~min}$, at which point benzyl bromide $(0.72 \mathrm{~g}, 0.5 \mathrm{ml}, 4.20 \mathrm{mmol})$ was added. After another $2 \mathrm{~h}$ of continuous heating, a second portion of benzyl bromide $(0.72 \mathrm{~g}, 0.5 \mathrm{ml}, 4.20$ mmol) was added. The heating was continued for $1 \mathrm{~h}$, after which the reaction mixture was let to stir overnight at room temperature. This yielded a white precipitate $\left(\mathrm{K}_{2} \mathrm{CO}_{3}\right)$ and a transparent liquid layer. The precipitate was filtered by suction and the solvent removed from the filtrate under reduced pressure. The obtained oily yellow layer was extracted with ethyl acetate $(40 \mathrm{ml})$ and the extract washed twice with water $(15 \mathrm{ml})$. The yellow organic layer was dried over sodium sulphate and concentrated by rotary evaporator under vacuum. The white precipitate was recrystallized from $\mathrm{CHCl}_{3}$ and the solid thus obtained collected by filtration, washed with chloroform, and dried under vacuum. Yield: $0.096 \mathrm{~g}$ (7.5 \%). ${ }^{1} \mathrm{H}$ NMR (DMSO-D $6,500 \mathrm{MHz}, 30$ $\left.{ }^{\circ} \mathrm{C}\right): \delta=11.68(\mathrm{~s}, 1 \mathrm{H}, \mathrm{H} 3), 8.33(\mathrm{~s}, 1 \mathrm{H}, \mathrm{H} 6), 7.29-7.38(\mathrm{~m}, 5 \mathrm{H}, \mathrm{ArH}), 4.88\left(\mathrm{~s}, 2 \mathrm{H}, \mathrm{CH}_{2}\right) .{ }^{13} \mathrm{C}$ NMR (126 MHz, DMSO-D $): \delta=160.9$ (1C, C4), 150.6 (1C, C2), 149.6 (1C, C6), 136.6 (1C, C8), 128.6 (2C, C9\&C13), 127.7 (1C, C11), 127.4 (2C, C10\&C12), 68.5 (1C, C5), 50.4 (1C, C7). ESI-TOF HRMS: $m / z$ [M-H] $]^{-}$calcd. for $\mathrm{C}_{11} \mathrm{H}_{9} \mathrm{IN}_{2} \mathrm{O}_{2} 326.96$, found 327.02. The analytical data correlates well with the literature data. ${ }^{12}$

Compound 3 (1-hexyl-5-iodopyrimidine-2,4(1H,3H)-dione): $5 \mathrm{IU}(0.92 \mathrm{~g}, 3.86 \mathrm{mmol})$ and $\mathrm{K}_{2} \mathrm{CO}_{3}(0.54 \mathrm{~g}, 3.86 \mathrm{mmol})$ was dissolved in dry DMF $(10 \mathrm{ml})$ in a round-bottomed flask. The reaction mixture was stirred and heated under $\mathrm{N}_{2}$ atmosphere at $80^{\circ} \mathrm{C}$. After 30 min, 1bromohexane $(1.27 \mathrm{~g}, 1.08 \mathrm{ml}, 7.72 \mathrm{mmol})$ was added into the reaction mixture and the heating was continued overnight. This gave a brownish-yellow oil that was concentrated under reduced pressure. The residue was extracted with ethyl acetate $(40 \mathrm{ml})$ and the extract washed twice with water $(15 \mathrm{ml})$. The organic layer was dried over sodium sulfate and concentrated by rotary evaporator under vacuum. The oily precipitate was crystallized from $\mathrm{CHCl}_{3}$ and the obtained 
white crystals were collected by filtration, washed with $\mathrm{CHCl}_{3}$, and dried under vacuum. Yield: 0.092 g (7.4 \%). ${ }^{1} \mathrm{H}$ NMR (500 MHz, DMSO-D $): \delta=11.53$ (s, 1H, H3), 8.20 (s, 1H, H6), 3.65 (t, 2H, H7), 1.52-1.59 (m, 2H, H8), 1.22-1.28 (m, 6H, H9-H11), 0.84-0.87 (t, 3H, H12). ${ }^{13} \mathrm{C}$ NMR (126 MHz, DMSO-D $): \delta=160.9$ (1C, C4), 150.6 (1C, C2), 149.9 (1C, C6), 67.7 (1C, C5), 47.7 (1C, C7), 30.7 (1C, C8), 28.3 (1C, C9), 25.3 (1C, C10), 21.8 (1C, C11), 13.8 (1C, C12). ESI-TOF HRMS: $m / z$ [M-H] $]^{-}$calcd. for $\mathrm{C}_{10} \mathrm{H}_{15} \mathrm{IN}_{2} \mathrm{O}_{2} 321.01$, found 321.03 .

Compound 4 (2,2'-(5-iodo-2,4-dioxopyrimidine-1,3(2H,4H)-diyl)bis(N,N,N-trimethylethanaminium) bromide): The preparation of 4 commenced by dissolving 5IU (0.92 g, $3.86 \mathrm{mmol})$ and $\mathrm{K}_{2} \mathrm{CO}_{3}(0.54 \mathrm{~g}, 3.86 \mathrm{mmol})$ in dry DMSO $(10 \mathrm{ml})$ in a round-bottomed flask. The reaction mixture was stirred and heated under $\mathrm{Ar}$ atmosphere at $50^{\circ} \mathrm{C}$. After $30 \mathrm{~min}$, (2bromoethyl)trimethylammonium bromide $(0.95 \mathrm{~g}, 3.86 \mathrm{mmol})$ was added into the reaction mixture and the heating was continued for 3 hours, at which point a second portion of (2bromoethyl)trimethylammonium bromide $(0.95 \mathrm{~g}, 3.86 \mathrm{mmol})$ was added. Heating the reaction mixture overnight gave a yellow solution with white solid which was poured hot in $50 \mathrm{ml}$ of cold acetone. The white solid was separated by filtration and recrystallized from a mixture of ethyl acetate/acetic acid (6:1) and dried under vacuum. Yield: $1.25 \mathrm{~g}(56.8 \%)$. ${ }^{1} \mathrm{H}$ NMR (500 MHz, $\left.\mathrm{D}_{2} \mathrm{O}\right): \delta=8.25(\mathrm{~s}, 1 \mathrm{H}, \mathrm{H} 6), 4.44-4.48(\mathrm{~m}, 2 \mathrm{H}, \mathrm{H} 13), 4.35-4.49(\mathrm{~m}, 2 \mathrm{H}, \mathrm{H} 7), 3.71-3.75(\mathrm{~m}, 2 \mathrm{H}$, H8), 3.59-3.62 (m, 2H, H14), 3.23 and 3.25 (2s, 18H, H10-H12\&H16-H18). ${ }^{13} \mathrm{C}$ NMR (126 MHz, $\left.\mathrm{D}_{2} \mathrm{O}\right): \delta=162.1(1 \mathrm{C}, \mathrm{C} 4), 151.5$ (1C, C2), 149.4 (1C, C6), 67.3 (1C, C5), 62.3 (1C, C8), 62.0 (1C, C14), 53.7 (3C, C10-C12), 53.5 (3C, C16-C18), 43.7 (1C, C7), $36.8(1 \mathrm{C}, \mathrm{C} 13) .{ }^{15} \mathrm{~N}$ NMR (51 MHz, $\left.\mathrm{D}_{2} \mathrm{O}\right): \delta=-224.1(\mathrm{~N} 3),-244.5$ (N1), -333.9 (N9\&N15). ESI-TOF HRMS: $m / z$ $\left[\mathrm{M}-2 \mathrm{Br}^{-}\right]^{2+}$ calcd. for $\mathrm{C}_{14} \mathrm{H}_{27} \mathrm{Br}_{2} \mathrm{IN}_{4} \mathrm{O}_{2} 205.06$, found 205.01. 


\section{Crystallizations}

Crystallizations of $\mathbf{1}$ in DMF, MeF and FA yielded solvated crystal structures $\mathbf{1} \cdot \mathrm{DMF}$ (1a), $\mathbf{1} \cdot \mathrm{MeF}(\mathbf{1 b})$ and $\mathbf{1}_{2} \cdot \mathrm{FA}(\mathbf{1 c})$. When the crystallization of 1 was carried out in DMF and in the presence of 1,4-diazabicyclo[2.2.2] octane (DABCO), a structure containing $\mathbf{1}, \mathrm{DABCOH}^{+}$and $\mathbf{1}^{-}$ was obtained (1d). Crystallization of 4 in FA gave $4 \cdot 2 \mathrm{H}_{2} \mathrm{O}$. Further information of crystallization studies are given in the Supporting Information.

\section{Single crystal X-ray diffraction studies}

Crystallographic data were collected at $123 \mathrm{~K}$ with a Bruker-Nonius Kappa CCD diffractometer with APEX II detector using graphite-monochromatized Mo-K $\alpha$ radiation $(\lambda=$ $0.71073 \AA$ ) except for 4a, for which with an Agilent SuperNova dual wavelength diffractometer equipped with Atlas $\mathrm{CCD}$ area detector with $\mathrm{Cu}-\mathrm{K} \alpha$ radiation $(\lambda=1.54184 \AA)$ were used. COLLECT/DENZO ${ }^{13 a, b}$ and CrysAlisPro ${ }^{13 c}$ software were utilized for the Kappa APEX II and Supernova instruments, respectively. The structures were solved by direct methods using SIR2004 ${ }^{13 \mathrm{~d}}$ program and full-matrix, least-squares refinements on $F^{2}$ were performed using SHELXL-97. ${ }^{13 e}$ The reflections were corrected for Lorenz polarization, and a multi-scan absorption correction (either SADABS ${ }^{13 f}$ or CrysAlisPro ${ }^{13 c}$ ) was applied. All hydrogen atoms bonded to carbon, except for those in formyl groups, were calculated to their idealized positions with isotropic temperature factors $\left[U_{\text {iso }}(\mathrm{H})\right.$ factors of 1.2 or 1.5 times $\left.U_{\text {eq }}(\mathrm{C})\right]$ and refined as riding atoms. Hydrogen atoms attached to nitrogen and oxygen atoms, or to formyl carbon atoms, were found from electron density maps and restrained to their ideal distances from the parent atoms $(0.88 \AA$ for $\mathrm{N}-\mathrm{H}, 0.84 \AA$ for $\mathrm{O}-\mathrm{H}$, and 0.95 for $\mathrm{C}-\mathrm{H})$ with $U_{\text {iso }}(\mathrm{H})$ factors of $1.2(\mathrm{~N}-$ $\mathrm{H}, \mathrm{C}-\mathrm{H})$ or $1.5(\mathrm{O}-\mathrm{H})$ times the parent atom factor. All figures were drawn with the Mercury 
program. ${ }^{13 \mathrm{~g}}$ Crystal data and refinement parameters are listed in Table 1, while full crystallographic data has been deposited to the Cambridge Crystallographic Data Centre (CCDC907245 (1c), 907246 (1b), 907247 (1a), 907248 (1d), and 907252 (4a)). These data can be obtained free of charge via www.ccdc.cam.ac.uk/data_request/cif.

Table 1. Crystallographic data of 1a-1d and 4a.

\begin{tabular}{|c|c|c|c|c|c|}
\hline & $\mathbf{1 a}$ & $\mathbf{1 b}$ & 1c & 1d & $4 a$ \\
\hline Empirical formula & $\mathrm{C}_{7} \mathrm{H}_{10} \mathrm{IN}_{3} \mathrm{O}_{3}$ & $\mathrm{C}_{6} \mathrm{H}_{8} \mathrm{IN}_{3} \mathrm{O}_{3}$ & $\mathrm{C}_{9} \mathrm{H}_{9} \mathrm{I}_{2} \mathrm{~N}_{5} \mathrm{O}_{5}$ & $\mathrm{C}_{14} \mathrm{H}_{18} \mathrm{I}_{2} \mathrm{~N}_{6} \mathrm{O}_{4}$ & $\mathrm{C}_{14} \mathrm{H}_{31} \mathrm{Br}_{2} \mathrm{IN}_{4} \mathrm{O}$ \\
\hline$M_{\mathrm{r}}$ & 311.08 & 297.05 & 521.01 & 588.14 & 606.15 \\
\hline Crystal size [mm] & $0.29 \times 0.14 \times 0.07$ & $0.22 \times 0.13 \times 0.06$ & $0.12 \times 0.10 \times 0.05$ & $0.32 \times 0.21 \times 0.14$ & $0.09 \times 0.08 \times 0.05$ \\
\hline Crystal system & Orthorhombic & Triclinic & Triclinic & Triclinic & Triclinic \\
\hline Space group & Pbca & $P-1$ & $P-1$ & $P-1$ & $P-1$ \\
\hline$a[\AA]$ & $6.9389(2)$ & $4.50630(10)$ & $7.0916(2)$ & $9.5362(2)$ & $7.7588(2)$ \\
\hline$b[\AA]$ & $9.1892(2)$ & $8.1457(3)$ & $8.0951(3)$ & $10.3117(3)$ & $11.0399(4)$ \\
\hline$c[\AA]$ & $31.8311(8)$ & $13.1203(5)$ & $13.7201(4)$ & $10.7814(3)$ & $13.3847(4)$ \\
\hline$\alpha\left[^{\circ}\right]$ & 90 & $80.312(2)$ & $75.532(2)$ & $103.448(2)$ & $80.870(2)$ \\
\hline$\beta\left[^{\circ}\right]$ & 90 & $84.365(2)$ & $88.011(2)$ & $94.687(2)$ & $81.662(2)$ \\
\hline$\gamma\left[{ }^{\circ}\right]$ & 90 & $80.214(2)$ & $70.744(2)$ & $113.607(2)$ & $79.795(3)$ \\
\hline$V\left[\AA^{3}\right]$ & 2029.64(9) & $466.63(3)$ & $719.01(4)$ & $926.46(4)$ & $1105.96(6)$ \\
\hline$Z$ & 8 & 2 & 2 & 2 & 2 \\
\hline$\rho_{\text {calc }}\left[\mathrm{Mgm}^{-3}\right]$ & 2.036 & 2.114 & 2.407 & 2.108 & 1.820 \\
\hline$\mu\left[\mathrm{mm}^{-1}\right]$ & 3.143 & 3.412 & 4.403 & 3.428 & 15.821 \\
\hline Max./min. transmission & $0.8100 / 0.4626$ & $0.8215 / 0.5207$ & $0.8099 / 0.6201$ & $0.6454 / 0.4067$ & $0.5051 / 0.3301$ \\
\hline$F(000)$ & 1200 & 284 & 488 & 564 & 596 \\
\hline$\theta$ range $\left[{ }^{\circ}\right]$ & 3.20 to 25.24 & 2.80 to 25.25 & 2.75 to 25.25 & 2.78 to 25.25 & 3.37 to 69.99 \\
\hline Completeness to $\theta[\%]$ & 99.6 & 99.7 & 99.5 & 99.6 & 99.0 \\
\hline Reflections collected & 3325 & 6128 & 4348 & 5615 & 6776 \\
\hline Data $^{a} /$ restraints/parameters & $1830 / 4 / 138$ & $1695 / 4 / 131$ & $2607 / 7 / 211$ & $3344 / 4 / 247$ & $4149 / 0 / 238$ \\
\hline$R_{\text {int }}$ & 0.0188 & 0.0322 & 0.0184 & 0.0193 & 0.0299 \\
\hline Reflections with $I>2 \sigma(I)$ & 1637 & 1587 & 2339 & 3069 & 4031 \\
\hline Final $R$ indices $[I>2 \sigma(I)]$ & $\begin{array}{l}R 1=0.0301 \\
\mathrm{w} R 2=0.0612\end{array}$ & $\begin{array}{l}R 1=0.0193 \\
\mathrm{w} R 2=0.0412\end{array}$ & $\begin{array}{l}R 1=0.0245 \\
\mathrm{w} R 2=0.0594\end{array}$ & $\begin{array}{l}R 1=0.0217 \\
\mathrm{w} R 2=0.0508\end{array}$ & $\begin{array}{l}R 1=0.0263 \\
\mathrm{w} R 2=0.0669\end{array}$ \\
\hline Final $R$ indices (all data) & $\begin{array}{l}R 1=0.0352 \\
\mathrm{w} R 2=0.0631\end{array}$ & $\begin{array}{l}R 1=0.0216 \\
\mathrm{w} R 2=0.0421\end{array}$ & $\begin{array}{l}R 1=0.0288 \\
\mathrm{w} R 2=0.0616\end{array}$ & $\begin{array}{l}R 1=0.0248 \\
\mathrm{w} R 2=0.0522\end{array}$ & $\begin{array}{l}R 1=0.0271 \\
\mathrm{w} R 2=0.0678\end{array}$ \\
\hline Goodness-of-fit on $F^{2}$ & 1.147 & 1.022 & 1.041 & 1.071 & 1.053 \\
\hline Largest diff. peak/hole & $0.820 /-0.545$ & $0.378 /-0.474$ & $0.962 /-0.481$ & $0.456 /-0.457$ & $0.596 /-1.043^{b}$ \\
\hline
\end{tabular}




\section{Spectroscopic measurements}

All NMR spectra were recorded with a Bruker Avance DRX 500 FT-NMR spectrometer at 303K. The chemical shifts are reported in ppm and referenced internally using the residual polar solvent resonances relative to tetramethylsilane (DMSO- $\mathrm{D}_{6} \delta=2.50, \mathrm{D}_{2} \mathrm{O} \delta=4.80$ ), except for the ${ }^{15} \mathrm{~N}$ data for which an external standard $\left(\mathrm{CH}_{3} \mathrm{NO}_{2}\right)$ was used. Mass spectrometric measurements were performed with a Micromass LCT time of flight (TOF) mass spectrometer with electrospray ionization (ESI).

\section{Computational details}

The geometries of the hydrogen and halogen bonded dimers 5-12 (see below) were optimized using the second-order local Møller-Plesset perturbation theory (LMP2) ${ }^{14}$ in conjunction with spin-component scaling (SCS) ${ }^{15}$ and density fitting (DF), ${ }^{16}$ yielding the DF-SCS-LMP2 method. This method was also used to calculate the interaction energies of the hydrogen and halogen bonded model dimers 5-12 and the potential energy surface scans of 6 and 11. Starting geometries for the model dimers were obtained from the acquired X-ray crystallographic data.

In all DF-SCS-LMP2 calculations, localized molecular orbitals were constructed using the Pipek-Mezey localization approach, ${ }^{17}$ whereas their corresponding domains were defined employing the Boughton and Pulay procedure. ${ }^{17 b}$ Domains were determined at large intermolecular distance and individual monomers were identified automatically in order to ensure interaction energies free of basis set superposition error (BSSE) and to obtain smooth potential energy surfaces. In the SCS correction, scaling factors $6 / 5$ and $1 / 3$ were used for the antiparallel and parallel spin components, respectively. 
Correlation consistent basis sets augmented with both polarization and diffusion functions, namely aug-cc-pVTZ, were used for all other nuclei except for iodine for which the small core ECP basis set aug-cc-pVTZ-PP was used. ${ }^{18}$ Auxiliary basis sets of triple- $\zeta$ valence quality were employed in the DF approximation to speed up all calculations. ${ }^{19}$ All quantum chemical calculations were done with the Molpro $2010.1^{20}$ program package; for visualization of optimized geometries, the program Mercury 3.1 was employed. ${ }^{13 g}$ 


\section{RESULTS AND DISCUSSION}

\section{CSD study}

As already mentioned in the introduction, only 26 structures containing a 5 IU unit were found in the CSD database and only 14 of them manifest XB interactions in which iodine functions as a XB donor. The analysis was restricted to $\mathrm{I} \cdots \mathrm{A}(\mathrm{A}=$ acceptor $)$ contacts that are shorter than 0.9 times the sum of van der Waals $(\mathrm{vdW})$ radii and have close to linear $\mathrm{C}-\mathrm{I} \cdots \mathrm{A}$ arrangement $(>$ $155^{\circ}$ ). The $\mathrm{XB}$ interactions found are listed in the Supporting Information along with the relevant contact parameters. As a whole, the CSD study clearly shows that the 5-iodouracil moiety can act as an XB donor, at least for carbonyl acceptors, although the presence of two carbonyls in the uracil ring often leads to other interactions such as hydrogen bonding.

\section{Structures of 5IU complexes}

During the design of the synthetic procedures, the solubility of $\mathbf{1}$ in different solvents was tested and DMF was found to be a good solvent for this particular system. Consequently, when a solution of 1 in DMF was left standing on an evaporation dish, well-formed colorless plate-like single crystals were obtained. The crystallographic analysis showed the crystals to be that of a DMF solvate of 1 (1a) in orthorhombic space group Pbca. The structural data shows that, in this instance, a short and linear $\mathrm{C}-\mathrm{I} \cdots \mathrm{O} \mathrm{XB}$ interaction binds the DMF molecules to 1 via the carbonyl oxygen (Figure 1). The $\mathrm{I} \cdots \mathrm{O}$ contact distance in $\mathbf{1 a}$ is $18 \%$ smaller than the sum of $\mathrm{vdW}$ radii for I and $\mathrm{O}$ atoms, which is of the same magnitude as the shortest XBs involving $\mathbf{1}$ reported in the CSD. ${ }^{21}$ 


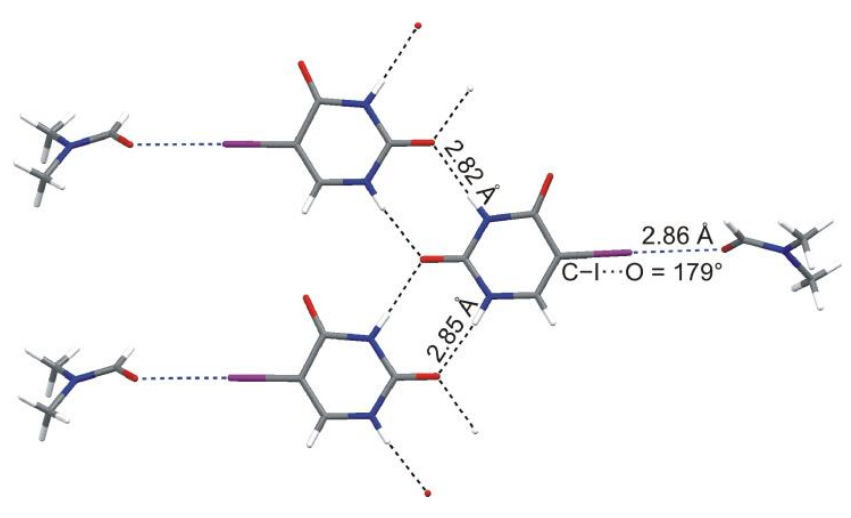

Figure 1. Three asymmetric units of 1 a showing the $\mathrm{XB}(\mathrm{C}-\mathrm{I} \cdots \mathrm{O}$, blue) and $\mathrm{HB}(\mathrm{N}-\mathrm{H} \cdots \mathrm{O}=\mathrm{C}$, black) interactions.

The structure of 1a inspired us to test other formamide solvents under the same conditions. To our surprise, a new polymorph of $\mathbf{1}$ was obtained when using DEF as the solvent. However, since this structure does not show any $\mathrm{XB}$ interactions, it is not discussed herein (see Supporting Information).

The crystallization of 1 from MeF yielded colorless plates which were shown to be a MeF solvate of 1 (1b) by X-ray diffraction. The structure of $\mathbf{1 b}$ has a triclinic $P-1$ space group and it shows similar but weaker $\mathrm{C}-\mathrm{I} \cdots \mathrm{O}$ XB interactions than 1a. The $\mathrm{I} \cdots \mathrm{O}$ distance is rather long and only a $10 \%$ reduction of the sum of vdW radii is observed. The apparently weaker XB in $\mathbf{1 b}$ can be explained with the influence of the "interfering" strong $\mathrm{N}-\mathrm{H} \cdots \mathrm{O}$ HB which affects the linearity of the $\mathrm{C}-\mathrm{I} \cdots \mathrm{O}$ unit (Figure 2). The hydrogen bonding pattern in $\mathbf{1 b}$ differs from that in 1a, which is expected due to the presence of a strong HB donor in MeF. 


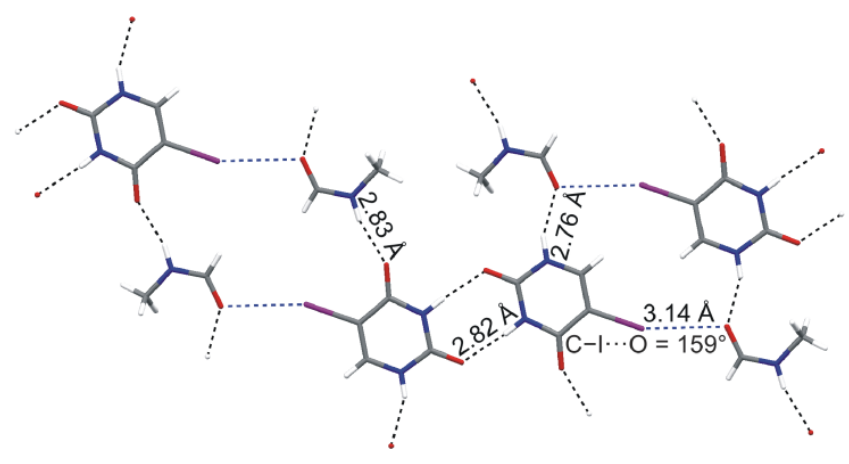

Figure 2. Four asymmetric units of $\mathbf{1 b}$ showing the $\mathrm{XB}(\mathrm{C}-\mathrm{I} \cdots \mathrm{O}$, blue $)$ and $\mathrm{HB}(\mathrm{N}-\mathrm{H} \cdots \mathrm{O}=\mathrm{C}$, black) interactions.

Crystallographic analysis of the colorless plates obtained from the FA solution of $\mathbf{1}$ showed it to contain two crystallographically independent molecules of $\mathbf{1}$ (denoted $A$ and $B$ ) and one molecule of FA (1c) in space group $P-1$. The two molecules of $\mathbf{1}$ display one $\mathrm{C}-\mathrm{I} \cdots \mathrm{O}$ interaction in which the carbonyl oxygen $\mathrm{O} 4$ of molecule $B$ acts as the $\mathrm{XB}$ acceptor for the iodine atom in molecule $A$ (Figure $3 \mathrm{a}$ ); the observed $\mathrm{I} \cdots \mathrm{O}$ distance in $\mathbf{1 c}$ is rather short $(17 \%$ reduction of the sum of $\mathrm{vdW}$ radii) though longer than in 1a. Interestingly, the iodine atom in molecule $B$ does not function as an XB donor. The single molecule of FA is hydrogen bonded to molecule $A$ by $\mathrm{R}_{2}^{2}(8)$ motif involving the $\mathrm{N} 3$ donor and $\mathrm{O} 2$ acceptor, ${ }^{22}$ and also to the $\mathrm{O} 4$ acceptor of molecule $B$. Molecule $A$ is also interacting with the solvent molecule in the adjacent asymmetric unit via its HB donor N1. Overall, 1c has an interesting packing (Figure 3b) in which molecules of $A$ and FA form layered stripe-like motifs that are linked together with Watson-Crick type HB contacts, ${ }^{23}$ formed by pairs of molecule $B$ (the angle between the $A$-FA and $B$ planes is ca. $72^{\circ}$ ). 

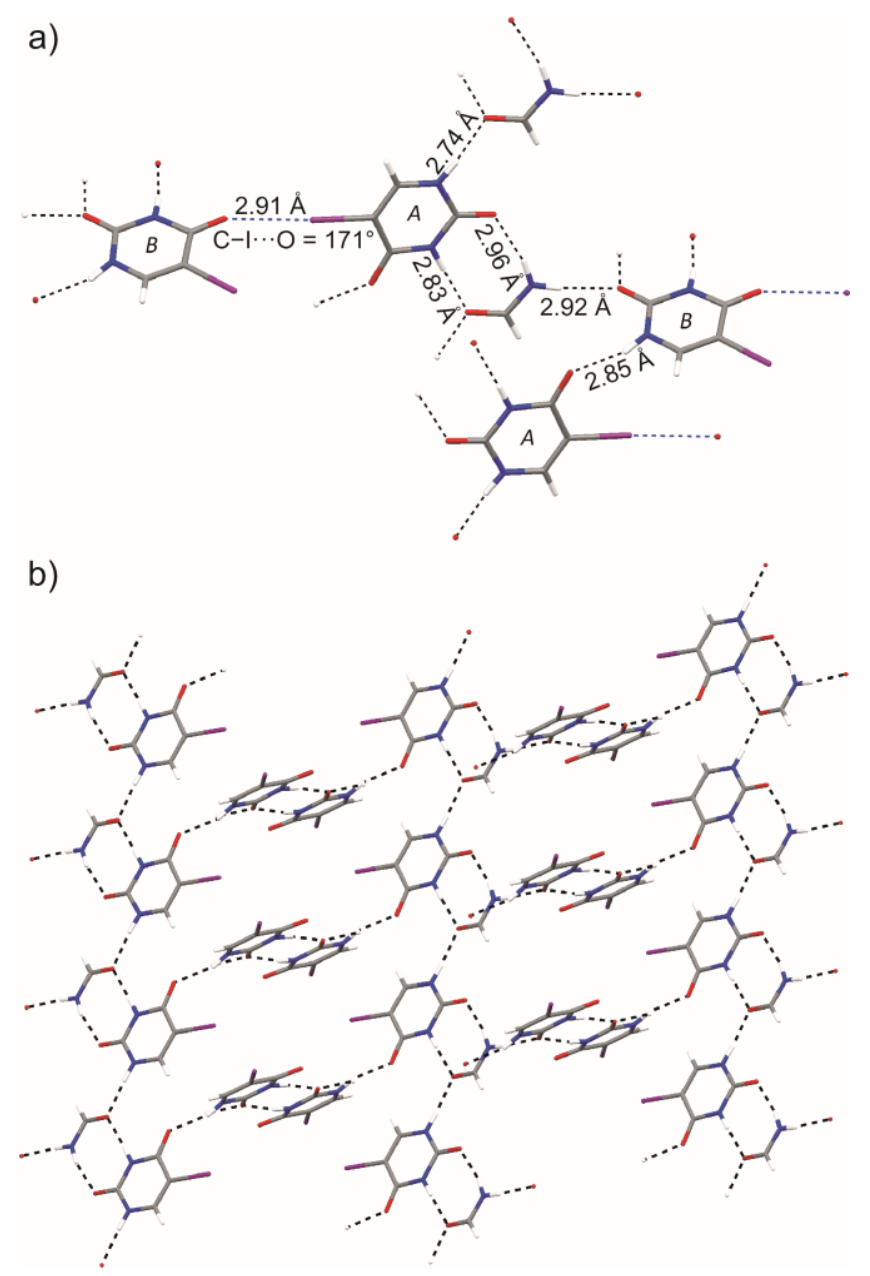

Figure 3. (a) Two asymmetric units of 1 c showing the $\mathrm{XB}(\mathrm{C}-\mathrm{I} \cdots \mathrm{O}$, blue $)$ and $\mathrm{HB}(\mathrm{N}-\mathrm{H} \cdots \mathrm{O}=\mathrm{C}$, black) interactions. (b) The packing of $\mathbf{1 c}$ in the solid state.

Almost all of our attempts to co-crystallize 5IU with different molecules failed (see Supporting Information) and crystalline material (colorless prisms, space group $P$-1) was only obtained from a mixture of 1 and DABCO in DMF. Subsequent crystallographic analysis gave a somewhat unexpected result: instead of a simple adduct $1 \cdot \mathrm{DABCO}$, the structure contains both neutral and deprotonated (N1) molecules of $\mathbf{1}$ (1d). A part of the sheet-like motif with HBs and XBs is shown in Figure 4. The $\mathrm{C}-\mathrm{I} \cdots \mathrm{O}$ XB interaction involves the anionic 5IU unit (donor) and the neutral molecule $\mathbf{1}$ (acceptor). A second XB is observed between the $\mathrm{C}-\mathrm{I}$ of $\mathbf{1}$ and the nitrogen 
atom of $\mathrm{DABCOH}^{+}$; $\mathrm{DABCOH}{ }^{+}$is also bound to $\mathbf{1}^{-}$via $\mathrm{HB}$ accepted by the carbonyl oxygen O2. A comparison of the observed XBs with the sum of $\mathrm{vdW}$ radii for the corresponding atoms shows ca. $15 \%$ reduction, indicative of moderate interaction strength.

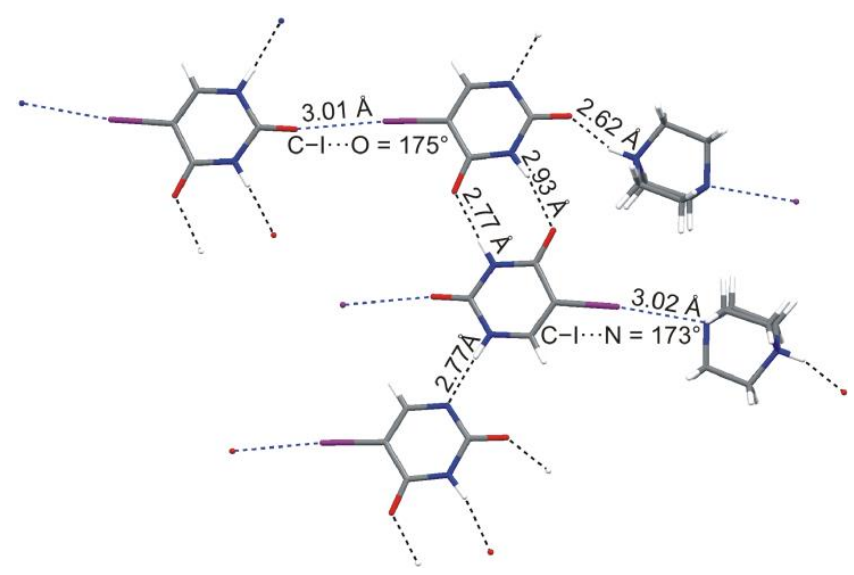

Figure 4. Part of the crystal structure of $1 \mathbf{d}$ showing XBs (blue) and HBs (black).

\section{Computational studies of 5IU complexes}

Even though the crystallographic evidence conclusively highlights the ability of $\mathbf{1}$ to act as a XB donor in 1a-d, high-level quantum chemical calculations for five simplified model dimers (59, Figure 5) were carried out in order to get insight into the strength of the observed interactions. For comparison purposes, hydrogen bonding interactions in four different model dimers involving 1 were also examined (10-12, Figure 5).

Because both XBs and HBs involve a varying degree of dispersion, electrostatics and charge transfer, not all quantum chemical methods can be used to describe them. ${ }^{24}$ One computational method that has been widely used for this purpose is the second-order Møller-Plesset perturbation theory (MP2). ${ }^{25}$ The MP2 method offers reasonable accuracy with relatively low 
computational cost, but unfortunately its standard implementation tends to overestimate the strength of both $\mathrm{XB}$ and HB. ${ }^{26,27}$ However, it was recently shown that the performance of MP2 with respect to XBs can be significantly improved by employing spin component scaling (SCS) ${ }^{15}$ in conjunction with large basis sets, and by removing the basis set superposition error (BSSE) using counterpoise correction. ${ }^{26 a, 28}$ The BSSE can also be removed with the help of localized orbital basis (LMP2), ${ }^{14}$ and the required CPU time can be significantly reduced with density fitting (DF). ${ }^{16}$ Consequently, the combined SCS-DF-LMP2 method was used in the current work in together with the aug-cc-pVTZ-(PP) basis sets. ${ }^{18}$
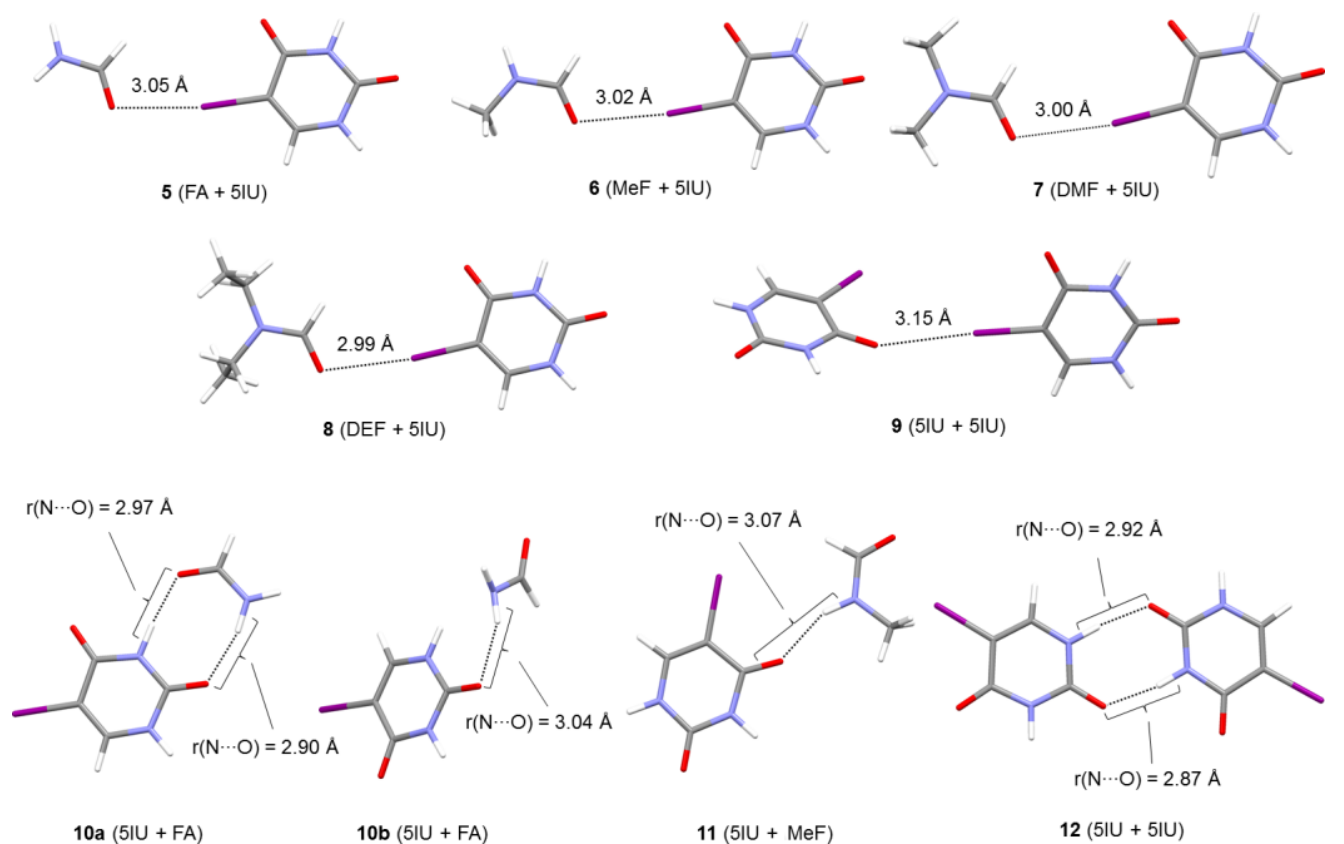

Figure 5. Optimized geometries of the simplified model systems 5-12.

The geometries of the dimers 5-12 were optimized in the gas phase at the SCS-DF-LMP2/augcc-pVTZ-(PP) level of theory; the XBs and N $\cdots \mathrm{O}$ distances in the optimized structures are listed in Table 2. As seen from the optimized structures, the calculated data are in good agreement with the experimental values, with the exception of $\mathbf{9}$ and $\mathbf{1 1}$ for which the deviation between the two 
sets of numbers is more than $0.15 \AA$. The differences between the experimental (solid state) and computational (gas phase) results originate most likely from the flatness of the potential energy surface with respect to both halogen and hydrogen bonds. ${ }^{11 \mathrm{~g}, 29}$ For instance, a $\pm 0.20 \AA$ displacement of the bonds from their calculated equilibrium distance weakens them by $1-2 \mathrm{~kJ}$ $\mathrm{mol}^{-1}$ (see Supporting Information). Such small energy differences are easily offset by crystal packing forces which the gas phase calculations naturally do not take into account. However, despite the small deviations between the experimental and optimized geometries, the calculated data can be safely used to evaluate the interaction energies of the HBs and XBs in 1a-d.

Table 2. Experimental and calculated $\mathrm{I} \cdots \mathrm{O}$ and $\mathrm{N} \cdots \mathrm{O}$ distances for halogen and hydrogen bonded complexes 5-12 and their interaction energies. ${ }^{a}$

\begin{tabular}{|c|c|c|c|c|}
\hline Compound & $\mathbf{I} \cdots \mathbf{O}[\AA ̊]$ & $\mathbf{N} \cdots \mathbf{O}[\AA ̊]$ & Exp. [Å] & $\Delta E\left[\mathrm{~kJ} \mathrm{~mol}^{-1}\right]$ \\
\hline 5 & 3.05 & - & - & v16.1 \\
\hline 6 & 3.02 & - & $3.136(2)$ & -18.4 \\
\hline 7 & 3.00 & - & $2.860(3)$ & -19.5 \\
\hline 8 & 2.99 & - & - & -20.2 \\
\hline 9 & 3.15 & - & $2.908(3)$ & -13.9 \\
\hline \multirow[t]{2}{*}{$10 \mathrm{a}$} & - & 2.97 & $2.962(5)$ & \multirow{2}{*}{-47.5} \\
\hline & - & 2.90 & $2.834(4)$ & \\
\hline $10 \mathrm{~b}$ & - & 3.04 & $2.923(4)$ & -27.2 \\
\hline 11 & - & 3.07 & $2.829(3)$ & -22.5 \\
\hline \multirow[t]{2}{*}{12} & - & 2.87 & $2.816(5)$ & \multirow{2}{*}{-54.8} \\
\hline & - & 2.92 & $2.851(4)$ & \\
\hline
\end{tabular}

The calculated interaction energies (at $0 \mathrm{~K}$ temperature, Table 2) show that the strength of the XBs in the model dimers 5-9 varies between -14.0 and $-20.0 \mathrm{~kJ} \mathrm{~mol}^{-1}$, which supports the statement that XBs involving 1 range from weak to moderate. For comparison, the ability of $\mathbf{1}$ to act as a $\mathrm{XB}$ donor has been briefly mentioned in another computational study in which the authors concluded that the interaction energies involving 1 settle between -5.0 and $-16.0 \mathrm{~kJ}$ 
$\mathrm{mol}^{-1}{ }^{6}$ The calculated interaction energies in Table 2 also show that XBs do not solely determine the solid state packing of 1a-d since all HBs are calculated to be of equal strength to the XBs (or stronger). Thus, it can be concluded that the calculated data shows the potential of 5IU unit as a XB donor of medium strength, and that the crystal packing observed for 1a-d results from delicate interplay between XBs and HBs.

\section{Structures of compounds 2-4}

Compounds $\mathbf{2}$ and $\mathbf{3}$ crystallized out only from DMF and DMSO solutions, respectively; X-ray diffraction quality crystals were also obtained from co-crystallization of $\mathbf{3}$ with DABCO in ethyl acetate. However, since none of these structures shows any XB interactions, they are not discussed further (see Supporting Information).

The crystallization of $\mathbf{4}$ from FA yielded colorless plates which were shown to be a dihydrate of 4 (4a) with a triclinic $P-1$ space group, though dry solvents were used throughout. The 5IU unit binds to a bromine anion via long $\mathrm{C}-\mathrm{I} \cdots \mathrm{Br}^{-} \mathrm{XB}$ interaction (Figure 6). The contact is approximately linear, but the reduction of the sum of $\mathrm{vdW}$ radii is only $11 \%$. The observed $\mathrm{I} \cdots \mathrm{Br}^{-}$interaction is perturbed by two $\mathrm{O}-\mathrm{H}^{\cdots} \mathrm{Br}^{-}$hydrogen bonds involving the co-crystallized water molecules. Apparently these HBs withdraw the anion away from the iodine and weaken the XB contact. It seems that $\mathbf{4}$, due to its several potential HB acceptors, absorbs water easily, which naturally influences the crystallization process and plays a dominant role in the packing of 4a. Interestingly, neither of the two carbonyl units on the uracil moiety functions as a HB acceptor, while four molecules of water and two $\mathrm{Br}^{-}$anions form a ring-like assembly connected via HBs, and the cations and anions bind to his assembly. Unfortunately, 4a does not show XB 
interactions involving oxygen or nitrogen acceptors, possibly due to the weak XB donor nature of 5IU.

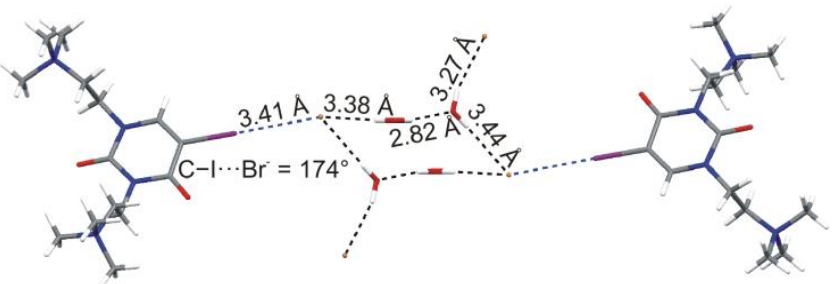

Figure 6. Non-covalent assembly in the crystal structure of $\mathbf{4 a}$, formed by two dications, four anions, and four water molecules, highlighting the relevant $\mathrm{XB}$ (blue) and $\mathrm{HB}$ (black) interactions. 


\section{CONCLUSIONS}

In the present study, we have investigated the halogen and hydrogen bonding properties of 5iodouracil $(5 \mathrm{IU}, \mathbf{1})$ and its derivatives (2-4) in polar solvents including formamides, DMSO, and water. In addition, co-crystallization experiments with 5IU and potential halogen bond acceptors were carried out. Nine new crystal structures were obtained of which five showed halogen bonding based on subsequent structural and computational analyses. Three of these structures contained solvated compounds (1a-c) while the remaining two were co-crystals (1d) or not solvated (4).

The obtained crystal structures, along with an analysis of the existing crystallographic data, demonstrate that the 5IU unit can act as a halogen bond donor, albeit a rather weak one. Halogen bonding interactions of the type $\mathrm{C}-\mathrm{I} \cdots \mathrm{O}$ were seen in four of the structures, while $\mathrm{C}-\mathrm{I} \cdots \mathrm{N}$ or $\mathrm{C}-\mathrm{I} \cdots \mathrm{Br}^{-}$interactions were observed in only instance each. The strongest interaction was seen in the structure of 1a which displayed the largest $(18 \%)$ reduction of the sum of van der Waals radii for the contact atoms. Typically, the observed reduction varied from 10 to $17 \%$, indicating weak to moderate interaction strength. Quantum chemical calculations on simplified model systems confirmed the attractive nature of the observed halogen bonds and showed that their interaction energy varies between 15 and $20 \mathrm{~kJ} \mathrm{~mol}^{-1}$. The $\mathrm{C}-\mathrm{I} \cdots \mathrm{N}$ type interaction observed for 1d is significantly shorter than the one currently reported in the literature, while the $\mathrm{I}^{\cdots} \mathrm{Br}^{-}$ interaction seen in 4a has not previously been demonstrated for 5IU or its derivatives. 


\section{ASSOCIATED CONTENT}

Supporting Information. Full crystallographic and computational information including details of crystallization and Cambridge Structural Database studies, full crystallographic data of synthesized compounds, and data from potential energy surface scans of model systems $\mathbf{6}$ and 11. This material is available free of charge via the Internet at http://pubs.acs.org.

\section{AUTHOR INFORMATION \\ Corresponding Author}

kari.t.rissanen@jyu.fi

\section{Address Information}

$\S$ Present address: Department of Biochemistry, University of Oulu, P.O. Box 3000, 90014 Oulu, Finland.

\section{Author Contributions}

The manuscript was written jointly and based on contributions from all authors. All authors have given approval to the final version of the manuscript.

\section{ACKNOWLEDGMENT}

The Academy of Finland (to K.R., grant nos. 122350, 140718, 265328, and 263256; to H. M. T, grant nos. 136929, 141000, and 253907) and the University of Jyväskylä (postdoctoral grant to A.V.) are gratefully acknowledged for financial support. 


\section{REFERENCES}

(1) Yang, Z.; Rodgers, M. T. J. Am. Chem. Soc. 2004, 126, 16217-16226.

(2) Camerman, N.; Trotter, J. Acta Crystallogr. 1965, 18, 203-211.

(3) Thomas, I. R.; Bruno, I. J.; Cole, J. C.; Macrae, C. F.; Pidcock, E.; Wood, P. A. J. Appl.

Crystallogr. 2010, 43, 362-366.

(4) (a) Sternglanz, H.; Freeman, G. R.; Bugg, C. E. Acta Crystallogr., Sect. B: Struct.

Crystallogr. Cryst. Chem. 1975, 31, 1393-1395. (b) Portalone, G. Acta Crystallogr., Sect. E: Struct. Rep. Online 2008, 64, o365.

(5) For examples of XBs involving 5IU in biological systems, see: (a) Auffinger, P.; Hays, F. A.; Westhof, E.; Ho, P. S. Proc. Natl. Acad. Sci. USA 2004, 101, 16789-16794. (b) Voth, A. R. Macromolecular Halogen Bonds, Ph.D. thesis, Oregon State University, 2007.

(6) XBs involving 5IU have also been analysed computationally, see Ref. 5a and Sarwar, M. G.; Dragisic, B.; Salsberg, L. J.; Gouliaras, C.; Taylor, M. S. J. Am. Chem. Soc. 2010, 132, 16461653.

(7) (a) Rissanen, K. CrystEngComm 2008, 10, 1107-1113. (b) Bertani, R.; Sgarbossa, P.; Venzo, A.; Lelj, F.; Amati, M.; Resnati, G.; Pilati, T.; Metrangolo, P.; Terraneo, G. Coord. Chem. Rev. 2010, 254, 677-695. (c) Cavallo, G.; Metrangolo, P.; Pilati, T.; Resnati, G.; Sansotera, M.; Terraneo, G. Chem. Soc. Rev. 2010, 39, 3772-3783. (d) Fourmigue, M. Curr. Opin. Solid State Mater. Sci. 2009, 13, 36-45. (e) Hathwar, V. R.; Gonnade, R. G.; Munshi, P.; Bhadbhade, M. M.; Guru Row, T. N. Cryst. Growth Des. 2011, 11, 1855-1862. (f) Troff, R.W.; Mäkelä, T.; Topić, F.; Valkonen, A.; Raatikainen, K.; Rissanen, K. Eur. J. Org. Chem. 2013, 1617-1637.

(8) (a) Erdélyi, M. Chem. Soc. Rev. 2012, 41, 3547-3557. (b) Beale, T. M.; Chudzinski, M. G.; Sarwar, M. G.; Taylor, M. S. Chem. Soc. Rev. 2013, 42, 1667-1680.

(9) (a) Scholfield, M. R.; Vander Zanden, C. M.; Carter, M.; Ho, P. S. Protein Sci. 2013, 22, 139-152. (b) Lu, Y. X.; Liu, Y. T.; Xu, Z. J.; Li, H. Y.; Liu, H. L.; Zhu, W. L. Expert Opin. Drug Discovery 2012, 7, 375-383. (c) Parisini, E.; Metrangolo, P.; Pilati, T.; Resnati, G.; Terraneo, G. Chem. Soc. Rev. 2011, 40, 2267-2278.

(10) Baldrighi, M.; Cavallo, G.; Chierotti, M. R.; Gobetto, R.; Metrangolo, P.; Pilati, T.; Resnati, G.; Terraneo, G. Mol. Pharmaceuticals 2013, 10, 1760-1722.

(11) (a) Tuikka, M.; Hirva, P.; Rissanen, K.; Korppi-Tommola, J.; Haukka, M. Chem. Commun. 2011, 47, 4499-4501. (b) Tuikka, M.; Niskanen, M.; Hirva, P.; Rissanen, K.; Valkonen, A.; Haukka, M. Chem. Commun. 2011, 47, 3427-3429. (c) Raatikainen, K.; Rissanen, K. CrystEngComm 2009, 11, 750-752. (d) Raatikainen, K.; Rissanen, K. Cryst. Growth Des. 2010, 10, 3638-3646. (e) Raatikainen, K.; Rissanen, K. CrystEngComm 2011, 13, 6972-6977. (f) Raatikainen, K.; Rissanen, K. Chem. Sci. 2012, 2, 1235-1239. (g) Peuronen, A.; Valkonen, A.; Kortelainen, M.; Rissanen, K.; Lahtinen, M. Cryst. Growth Des. 2012, 12, 4157-4169. (h) Troff, R. W.; Mäkelä, T.; Topić, F.; Valkonen, A.; Raatikainen, K.; Rissanen, K. Eur. J. Org. Chem. 2013, 1617-1637. (i) Raatikainen, K.; Cavallo, G.; Metrangolo, P.; Resnati, G.; Rissanen, K.; Terraneo, G. Cryst. Growth Des. 2013, 13, 871-877.

(12) Prachayasittikul, S.; Sornsongkhram, N.; Pingaew, R.; Worachartcheewan, A.; Ruchirawat, S.; Prachayasittikul, V. Molecules 2009, 14, 2768-2779.

(13) (a) COLLECT; Bruker AXS Inc.: Madison, WI, 2004. (b) Otwinowski Z.; Minor, W. Methods Enzymol. 1997, 276, 307-326. (c) CrysAlisPro, Version 1.171.36.20; Agilent Technologies Ltd: Yarnton, England, 2011. (d) Burla, M. C.; Caliandro, R.; Camalli, M.; 
Carrozzini, B.; Cascarano, G. L.; De Caro, L.; Giacovazzo, C.; Polidori, G.; Spagna, R. J. Appl. Crystallogr. 2005, 38, 381-388. (e) Sheldrick, G. M. Acta Crystallogr., Sect. A: Found.

Crystallogr. 2008, 64, 112-122. (f) Sheldrick, G. M. SADABS; University of Göttingen:

Göttingen, Germany, 1996. (g) Macrae, C. F.; Bruno, I. J.; Chisholm, J. A.; Edgington, P. R.; McCabe, P.; Pidcock, E.; Rodriguez-Monge, L.; Taylor, R.; van de Streek, J.; Wood, P. A. J. Appl. Crystallogr. 2008, 41, 466-470.

(14) Werner, H.; Pflüger, K. in "On the selection of domains and orbital pairs in local correlation treatments", Annual Reports in Computational Chemistry; Spellmeyer, D. C. (Ed.), Elsevier:

New York, USA, 2006, pp. 53-80.

(15) Grimme, S. J. Chem. Phys. 2003, 118, 9095-9102.

(16) (a) Schrader, D. M.; Prager, S. J. Chem. Phys. 1962, 37, 1456-1460. (b) Werner, H.-J.;

Manby, F. R.; Knowles, P. J. Chem. Phys. 2003, 118, 8149-8160.

(17) (a) Pipek, J.; Mezey, P. G. J. Chem. Phys. 1989, 90, 4916-4926. (b) James, W.; Boughton, P. P. J. Comput. Chem. 1993, 14, 736-740.

(18) (a) Dunning, T. H. Jr. J. Chem. Phys. 1989, 90, 1007-1023. (b) Kendall, R. A.; Dunning, T. H., Jr.; Harrison, R. J. J. Chem. Phys. 1992, 96, 6796-6806. (c) Peterson, K. A.; Shepler, B. C.; Figgen, D.; Stoll, H. J. Phys. Chem. A 2006, 110, 13877-13883.

(19) (a) Weigend, F.; Köhn, A.; Hättig, C. J. Chem. Phys. 2002, 116, 3175-3183. (b) Hättig, C. Phys. Chem. Chem. Phys. 2005, 7, 59-66. (c) Hellweg, C.; Hättig, C.; Höfener, S.; Klopper, W. Theor. Chem. Acc. 2007, 117, 587-597.

(20) Werner, H.-J.; Knowles, P. J.; Manby, F. R.; Schütz, M.; Celani, Knizia, G.; P.; Korona, T.; Lindh, R.; Mitrushenkov, A.; Rauhut, G.; Adler, T. B.; Amos, R. D.; Bernhardsson, A.; Berning, A.; Cooper, D. L.; Deegan, M. J. O.; Dobbyn, A. J.; Eckert, F.; Goll, E.; Hampel, C.;

Hesselmann, A.; Hetzer, G.; Hrenar, T.; Jansen, G.; Köppl, C.; Liu, Y.; Lloyd, A. W.; Mata, R. A.; May, A. J.; McNicholas, S. J.; Meyer, W.; Mura, M. E.; Nicklaß, A.; Palmieri, P.; Pflüger, K.; Pitzer, R.; Reiher, M.; Shiozaki, T.; Stoll, H.; Stone, A. J.; Tarroni, R.; Thorsteinsson, T.; Wang, M.; Wolf, A. MOLPRO, version 2010.1; http://www.molpro.net.

(21) De Winter, H. L.; Blaton, N. M.; Peeters, O. M.; De Ranter, C. J.; Van Aerschot, A.; Herdewijn, P. Acta Crystallogr., Sect. C: Cryst. Struct. Commun. 1991, 47, 2245-2247 (22) Bernstein, J.; Davis, R. E.; Shimoni, L.; Chang, N.-L. Angew. Chem. Int. Ed. Engl. 1995, $34,1555-1573$.

(23) Murata, T.; Umemoto, Y.; Miyazaki, E.; Nakasuji, K.; Morita, Y. CrystEngComm 2012, 14, 6881-6887.

(24) (a) Arunan, E.; Desiraju, G. R.; Klein, R. A.; Sadlej, J.; Scheiner, S.; Alkorta, I.; Clary, D. C.; Crabtree, R. H.; Dannenberg, J. J.; Hobza, P.; Kjaergaard, H. G.; Legon, A. C.; Mennucci, B.; Nesbitt, D. J. Pure Appl. Chem. 2011, 83, 1637-1641. (b) Metrangolo, P.; Resnati, G.; Desiraju, G. R.; Ho, P. S.; Kloo, L.; Legon, A. C.; Marquardt, R.; Politzer, P.; Rissanen, K. "Project: Categorizing Halogen Bonding and Other Noncovalent Interactions Involving Halogen Atoms. http://www.halogenbonding.eu/ (accessed on February, 2013).

(25) Møller, C.; Plesset, M. S. Phys. Rev. 1934, 46, 618-622.

(26) (a) Kozuch, S.; Martin, M. L. J. Chem. Theory Comput. 2013, 9, 1918-1931. (b) Zhao, Y.; Truhlar, D. G. J. Phys. Chem. A 2005, 109, 6624-6627.

(27) It should be noted that MP2 does not always overestimate the interaction strength of hydrogen bonds; the difference between high-level coupled cluster (CCSD(T)) and MP2 interaction energies can sometimes be negative. For some examples, see: (a) Jurečka, P.; Hobza 
P. J. Am. Chem. Soc. 2003, 125, 15608-15613. (b) Dąbkowska, I.; Gonzalez, H. V.; Jurečka, P.; Hobza, P. J. Phys. Chem. A 2005, 109, 1131-1136.

(28) There are some reports which indicate that the spin component scaling does not necessarily improve the description of hydrogen bonds with MP2. For some examples, see: (a) Bachorz, R. A.; Bischoff, F. A.; Höfener, S.; Klopper, W.; Ottiger, P.; Leist, R.; Frey, J. A.; Leutwyler, S. Phys. Chem. Chem. Phys. 2008, 10, 2758-2766. (b) Antony, J.; Grimme, S. J. Phys. Chem. A 2007, 111, 4862-4868.

(29) (a) Tsuzuki, S.; Uchimaru, T.; Wakisaka, A.; Onoc, T.; Sonodad, T. Phys. Chem. Chem. Phys. 2013, 15, 6088-6096. (b) Hill, G. J.; Hu, X. Chem.-Eur. J. 2013, 19, 3620-3628. (c) Voth, A. R.; Khuu, P.; Oishi, K.; Ho, P. S. Nature 2009, 1, 77-79. 